\title{
Se former à distance de manière collaborative : Le cas du réseau Twictée
}

\author{
Distance learning in a collaborative manner: \\ The case of the Twictée network
}

\section{Aprendizaje a distancia de manera colaborativa: El caso de la red Twictée}

Georges Ferone, maître de conférences

Université Paris-Est Créteil, France

georges.ferone@u-pec.fr

Jacques Crinon, professeur des universités

Université Paris-Est Créteil, France

jacques.crinon@u-pec.fr

RÉSUMÉ

Les réseaux connectés d'enseignants se sont fortement développés ces dernières années et ils se définissent aujourd'hui comme contribuant à la formation et au développement professionnel de leurs membres. Leurs effets diffèrent toutefois fortement selon le type de collectif et selon les individus. Cette étude concerne le réseau Twictée qui regroupe plusieurs centaines d'enseignants francophones de l'élémentaire et du collège mettant en œuvre un dispositif innovant d'enseignement de l'orthographe et échangeant à distance sur cet enseignement. L'analyse porte sur des entretiens menés avec dix-neuf enseignants sur deux années scolaires. Elle montre que le réseau Twictée peut être défini comme une communauté d'apprentissage professionnelle et elle met en évidence quatre profils de participants selon la nature et l'intensité de leur engagement. Les dynamiques de changement, quand elles existent, touchent surtout aux modes d'organisation pédagogique et à l'intégration $\mathrm{du}$ numérique dans les pratiques d'enseignement. Des changements identitaires se produisent chez les acteurs les plus engagés de la communauté.

Mots-clés : collaboration à distance, communauté en ligne, développement professionnel des enseignants, identité professionnelle, interactions, savoirs professionnels 
Connected teacher networks have grown significantly in recent years and define themselves as contributing to their members' professional development. However, their effects differ greatly depending on the collective and according to the individuals. This study examined the Twictee network, which brings together several hundred French-speaking elementary and secondary school teachers implementing an innovative system for teaching spelling and exchanging remotely. The analysis covers interviews conducted with nineteen teachers over two school years. Results show that the Twictée network can be defined as a professional learning community and highlights four participants' profiles according to the nature and intensity of their involvement. The dynamics of change, when they exist, relate mainly to methods of educational organization and the integration of digital technology into teaching practices. Identity changes are occurring among the most committed actors in the community.

Keywords: distance collaboration, online community, teacher professional development, professional identity, interactions, professional knowledge

RESUMEN

Las redes conectadas de profesores se han desarrollado significativamente en los últimos años y hoy se consideran contribuyentes del desarrollo profesional de sus miembros. Sin embargo, sus efectos difieren mucho según el tipo de colectivo y según los individuos. Este estudio se refiere a la red Twictée que reúne a centenares de profesores francófonos de primaria y de secundaria implementando un dispositivo innovador para enseñar ortografía e intercambiar a distancia sobre este tipo de enseñanza. El análisis trata entrevistas con diecinueve maestros durante dos años escolares. Muestra que la red Twictée se puede definir como una comunidad profesional de aprendizaje y destaca cuatro perfiles de participantes de acuerdo con la naturaleza y la intensidad de su compromiso. La dinámica del cambio, cuando existe, se relaciona principalmente con los modos de organización pedagógica y con la integración de la tecnología digital en las prácticas de enseñanza. Se observan cambios de identidad en los actores más comprometidos de la comunidad.

Palabras clave: colaboración a distancia, comunidad en línea, desarrollo profesional de los profesores, identidad profesional, interacciones, conocimientos profesionales

Les collectifs connectés d'enseignants existent depuis l'apparition d'Internet (Henri et Pudelko, 2002) et ils se sont fortement développés avec l'apparition des réseaux sociaux. Ces communautés enseignantes se positionnent aujourd'hui comme contribuant activement à la formation. Elles affirment volontiers que les interactions entre leurs membres et le partage de ressources favorisent l'apprentissage collaboratif et " constituent un puissant moteur de coformation » (Sésamath, 2020) et de développement professionnel. Dans ce contexte, nous analysons un collectif en ligne, le réseau Twictée. Celui-ci promeut un dispositif collaboratif d'enseignement et d'apprentissage de l'orthographe nommé « dispositif Twictée »; il est issu de l'initiative de praticiens et dispose d'un fort soutien institutionnel. II s'est rapidement développé en quelques années et il revendique de manière explicite des effets sur la formation des participants. Nous cherchons à identifier les conditions d'engagement et de participation de ses membres ainsi que les effets de cet engagement perçus par les acteurs. Avant de présenter cette étude, nous précisons la notion de développement professionnel enseignant, les conditions susceptibles de le favoriser et rappelons les principaux résultats antérieurs de recherche relatifs aux effets de la participation à un collectif en ligne. 


\title{
Développement professionnel, réseaux, communautés d'apprentissage
}

\author{
Le développement professionnel des enseignants (DPE)
}

Le développement professionnel touche aux savoirs disciplinaires, curriculaires et d'expérience (Tardif, Lessard et Lahaye, 1991), aux savoir-agir stratégiques (Uwamariya et Mukamurera, 2005), mais également à l'identité (Kaddouri, 2011; Marcel, 2005), à l'engagement individuel et collectif (Lameul, Peltier et Charlier, 2014) ainsi qu'aux progrès dans les apprentissages et à la réussite scolaire des élèves (Dionne, Lemyre et Savoie-Zajc, 2010; Leclerc et Labelle, 2013). Au regard de ces travaux, nous considèrerons le développement professionnel comme un processus individuel, structuré socialement, de changement de pratiques et d'acquisition progressive de compétences reconnues par la personne ellemême et par la communauté professionnelle dans laquelle la personne prend une part active et s'engage en vue d'améliorer l'apprentissage et la réussite scolaire des élèves.

Que savons-nous sur le lien entre la participation à un collectif en ligne et le développement professionnel? Dès la fin des années 90, des recherches ont concerné les effets des échanges électroniques sur les apprenants (Koschmann, 1996; Scardamalia et Bereiter, 1994) et sur les enseignants (Laferrière, 2001). Avec l'apparition des réseaux sociaux, les travaux se sont multipliés en Amérique du Nord où la notion de communauté éducative constitue une valeur importante, mais également en Europe (Daele, 2013). Wenger, Trayner et De Laat (2011) distinguent deux grandes catégories de collectifs en ligne, les réseaux d'apprentissage professionnels (Professional Learning Networks, PLN) et les communautés d'apprentissage professionnelles (Professional Learning communities, PLC), en considérant que ce qui distingue les PLC des PNL, c'est le développement, dans les secondes, de valeurs et d'une identité partagée autour d'un sujet ou d'un ensemble de défis. Quentin (2014) utilise les métaphores du bac à sable et de la ruche pour caractériser deux types différents de collectifs en ligne, qu'elle situe aux extrémités d'un continuum. Les collectifs représentatifs du type bac à sable seraient à classer dans les PNL, ceux du type de la ruche, dans les communautés'.

\section{Effets des réseaux d'apprentissage professionnels sur le DPE}

Trust, Krutka et Carpenter (2016) postulent que les réseaux professionnels d'enseignants sont des systèmes d'interactions complexes et personnalisés, composés de personnes, de ressources et d'outils numériques qui favorisent l'apprentissage continu et la croissance professionnelle. Caviale et Bruillard (2009) ainsi que Vangrieken, Meredith, Packer et Kyndt (2017) relativisent toutefois ces effets et montrent que les réseaux qui répondent à une commande institutionnelle et sont pilotés par un expert favorisent la transmission d'informations institutionnelles, mais ne constituent pas des lieux véritables de collaboration ou de discussion sur les pratiques. Ces réseaux fonctionnent avec des règles souples et souvent implicites; ce sont des espaces dans lesquels une minorité d'enseignants donnent à voir leur pratique professionnelle. Dans ce type de collectif, Quentin (2014) observe qu'il n'y a pas de véritable collaboration, mais une juxtaposition d'idées, sans tâche collaborative ni langage partagé par le groupe. Dans une autre recherche, Quentin (2019) analyse des comptes Twitter d'enseignants et conclut que le fonctionnement de Twitter, caractérisé par la brièveté des messages, par l'autopromotion et par la présence de trolls ${ }^{2}$, ne favorise pas la construction collective d'idées ou de connaissances.

\footnotetext{
${ }^{1}$ Quentin (2014) rappelle toutefois que « l'étude des réseaux professionnels en ligne est une affaire délicate (car) il s'agit en effet d'organisations complexes, protéiformes et en évolution perpétuelle » (p. 2). Baron et Zablot (2017) mentionnent qu'il existe une perméabilité entre ce qui est de l'ordre de la communauté et de ce qui relève du réseau social.

${ }^{2}$ Terme emprunté à la mythologie scandinave : être malveillant. Par extension, utilisateurs qui critiquent violemment d'autres utilisateurs sur les réseaux sociaux.
} 
Selon les études, les effets des réseaux d'apprentissage professionnels apparaissent très variables. Ils varient également d'un participant à l'autre. Ils concernent aussi bien le soutien moral et affectif que l'apport de ressources ou de stratégies d'enseignement. Cette participation aurait toutefois des effets limités sur le développement professionnel de ses membres du fait de l'absence d'élaboration collective ou de discussions approfondies sur les pratiques ou sur les apprentissages des élèves.

\section{Effets des communautés d'apprentissage professionnelles sur le DPE}

Les travaux sur les communautés d'apprentissage se réfèrent aux théories de Wenger (1998), pour qui une communauté de pratique est un groupe qui interagit, apprend ensemble, construit des relations et, à travers ces processus, développe un sentiment d'appartenance et d'engagement mutuel.

Vangrieken, Meredith, Packer et Kyndt (2017) identifient deux types de communautés susceptibles de favoriser le DPE : la communauté orientée et la communauté formative. Les communautés orientées fixent préalablement les buts et l'organisation de travail. Dans ce type de collectif, les objectifs sont de permettre d'échanger sur les conceptions et les pratiques, de partager des informations et des ressources, et de discuter des stratégies d'enseignement et des apprentissages de leurs élèves en vue de les faire progresser. À l'inverse, dans les communautés formatives, les objectifs et les modalités de travail s'élaborent et se négocient en fonction des attentes et des besoins des membres.

Proches des communautés orientées, les collectifs qu'on pourrait décrire comme appartenant au type ruche, comme Sésamath ${ }^{3}$ (Quentin, 2014), imposent des règles strictes de fonctionnement; les tâches sont clairement réparties et planifiées entre les membres qui doivent respecter leurs engagements. Contrairement au " bac à sable » où la promotion des individus est importante, ici c'est le groupe qui prime.

La participation à ces communautés d'apprentissage « orientées » favoriserait la motivation, l'estime et la confiance en soi, le sentiment d'efficacité personnelle et l'engagement dans le métier (Peters et SavoieZajc, 2013), sur la durée (Lantheaume, 2016). Elle apporterait un soutien émotionnel important et un encouragement à la prise de risque (Lantz-Andersson, Lundin et Selwyn, 2018). Elle enrichirait le répertoire des stratégies d'enseignement plus centrées sur les élèves et permettrait un meilleur alignement entre le curriculum réel et le curriculum caché (Vangrieken et al., 2017). L'engagement dans de telles communautés favoriserait donc le développement professionnel.

\section{Communautés et obstacles au DPE}

D'autres études expriment à l'inverse des réserves sur la capacité de ces communautés à favoriser le développement professionnel des enseignants.

Le premier obstacle est relatif à la nature et à l'intensité de la participation, qui conditionnent le succès d'une communauté selon Wenger (1998). Les travaux pointent de nombreux freins, liés à la taille de la communauté (Drot-Delange, 2001), aux statuts des membres (Caviale et Bruillard, 2009) et au manque d'ancienneté dans la communauté (Audran et Simonian, 2009). Le second obstacle est relatif à la difficulté des enseignants à exposer leurs pratiques et à développer des interactions à partir de celles-ci.

\footnotetext{
${ }^{3}$ https://www.sesamath.net/
} 
Le processus de développement professionnel en effet va de pair avec un retour réflexif, individuel et collectif sur la pratique, avec la mise en mots de cette pratique et sa conceptualisation (Pastré, Mayen et Vergnaud, 2006), voire avec des controverses dialogiques (Clot, 2005). Cela suppose pour les enseignants de s'engager dans des collectifs où le cœur des échanges porte sur leurs pratiques réelles, sur les apprentissages, les réussites et les difficultés qu'ils identifient chez leurs élèves. II s'agit de rendre visibles des pratiques généralement invisibles, notamment par le biais de préparations concertées et d'analyses conjointes de productions des élèves (Dupriez, 2015). Champy-Remoussenard (2014) expose le dilemme auquel sont confrontés les enseignants à ce sujet. Seuls dans leur classe, ils souffrent d'un déficit de visibilité et de reconnaissance de leur métier; montrer son travail répond donc à une quête de reconnaissance et de légitimité, mais en même temps, ce regard extérieur constitue une menace potentielle, une intrusion dans l'intimité du travail. Ce regard est donc recherché et craint à la fois. Wells et Feun (2007) constatent ainsi qu'il est difficile pour les enseignants d'aller au-delà du simple partage d'idées et de ressources et d'engager des discussions approfondies sur leurs stratégies d'enseignement et sur les apprentissages de leurs élèves. Macià et García (2016) mettent également en évidence la réticence de certains enseignants à collaborer ou à participer à des communautés en ligne par peur d'être critiqués, par manque d'expérience ou par insécurité à exposer leurs idées.

À partir de ces pratiques, des interactions devraient s'engager afin "de viser un "transpersonnel" irréductible à une collection de recettes intransmissibles ou à de bonnes pratiques imposées " (Rayou, 2018, p. 99). Or, les chercheurs observent que les échanges dans les collectifs en ligne ont le plus souvent des effets de normalisation (Beauné et al., 2019). Lantz-Andersson, Lundin et Selwyn (2018) constatent que, si les communautés sont censées favoriser des formes de réflexion et d'auto-analyse plus approfondies qu'en présence grâce à la confrontation des points de vue et aux échanges écrits, la réalité qu'ils décrivent est en fait bien différente. Leur revue de cinquante-deux études montre que les interactions restent le plus souvent superficielles, que les contenus ne sont jamais véritablement abordés. Ils s'interrogent de ce fait sur la valeur de discussions trop courtoises où l'esprit critique est absent. Bergviken Rensfeldt, Hillman et Selwyn (2018) ont analysé une communauté Facebook " classe inversée » (treize mille membres). Ils soulignent que celle-ci rompt le sentiment d'isolement, accroit le sentiment d'appartenance et procure une source d'information, mais ils montrent également qu'elle sert en particulier à l'autopromotion d'une minorité d'enseignants, qu'elle encourage la réutilisation de ressources plutôt que leur création, qu'elle est peu démocratique et favorise la diffusion d'une pensée uniforme.

Les différents travaux sur la relation entre communautés et développement professionnel montrent donc à la fois des potentiels et des obstacles, mais aussi la complexité de cet objet d'étude. Qu'en est-il pour le dispositif Twictée qui permettrait selon ses initiateurs de se former « de manière collaborative, sans injonction, sans préconisation par le biais de la curiosité partagée ${ }^{4}$ ॥? Pour répondre à cette question, nous avons mené, avec dix-neuf enseignants participant au dispositif, une série de trois entretiens sur une période de deux années scolaires. Nous décrivons dans un premier temps le dispositif, puis nous analysons les entretiens, du point de vue des conditions de leur participation au collectif Twictée et des effets perçus sur leur développement professionnel.

\footnotetext{
${ }^{4}$ https://www.twictee.org/twictee/
} 


\section{Twictée : un dispositif collaboratif d'enseignement et d'apprentissage de l'orthographe}

Twictée ${ }^{5}$, contraction des mots "Twitter » et « dictée », est un dispositif collaboratif d'enseignement de l'orthographe qui favorise, à partir d'échanges entre classes distantes via Twitter, l'élaboration par les élèves d'une correction argumentée des erreurs des partenaires et de leurs propres erreurs ${ }^{6}$. Ce dispositif a été créé en 2013 par deux enseignants du premier degré, Fabien Hobart et Régis Forgione; il regroupait en 2019 environ huit cents classes. Twictée s'est constituée en une association (l'adhésion ne conditionne pas la participation) qui ambitionne de créer « une dynamique de partage et de collaboration qui fédère les membres du collectif ». Cette collaboration peut se dérouler à distance (en utilisant Twitter, slack, messagerie) et en présence (réunions de l'association, université d'été ${ }^{7}$, cafés Twictée ${ }^{8}$ ).

Comment caractériser ce collectif enseignant? Selon les principales typologies relatives aux collectifs connectés, Twictée pourrait être classée dans les communautés d'apprentissage qui visent le partage de savoirs et le soutien entre collègues (Dionne, Lemyre et Savoie-Zajc, 2010), ou dans les communautés d'apprentissage professionnelles qui visent l'amélioration continue des résultats des élèves par le développement professionnel de ses membres (Leclerc et Labelle, 2013). Les trois conditions d'apprentissage définies par Wenger (1998) apparaissent :

- un engagement mutuel (Twictée fédère un collectif très actif et se développe de manière exponentielle depuis sa création),

- une entreprise commune (l'organisation évolue chaque année et les membres du réseau sont à l'origine de nombreuses créations de projets sur Twitter ${ }^{9}$ ),

- un répertoire partagé (de nombreux outils ont été créés, \#DicoBalises, rallyes twoutils et les membres partagent un vocabulaire commun propre au réseau, plombier, twictonaute, twoutils).

On retrouve également dans Twictée certaines caractéristiques de la « ruche » décrite par Quentin (2014), les règles, le calendrier de fonctionnement et la répartition des tâches sont définis précisément et les participants s'engagent à respecter la charte du Twictonaute ${ }^{10}$.

Ce réseau semble donc offrir un réel potentiel de développement professionnel au sens où nous l'avons défini. La participation à ce collectif pourrait permettre d'innover dans ses pratiques, d'acquérir des compétences en approfondissant les savoirs disciplinaires et d'expérience, notamment dans l'enseignement de l'orthographe, de renforcer l'engagement dans le métier et l'attention sur les progrès et la réussite scolaire des élèves. Qu'en est-il pour les enseignants que nous avons interrogés?

\footnotetext{
${ }^{5}$ Twictée est à la fois un collectif enseignant, une association, un dispositif d'enseignement de l'orthographe et une forme de dictée. Nous utiliserons la majuscule quand il s'agit du réseau des participants et du dispositif pédagogique, et la minuscule quand il s'agit des productions des dictées.

${ }^{6}$ Une twictée se déroule selon quatre phases : 1. Dictée individuelle sans préparation, dictée négociée par groupe, envoi des dictées (tweets) à la classe correspondante; correction de la dictée en collectif; 2 . Réception des dictées des correspondants, repérage des erreurs, rédaction des twoutils (explication de l'erreur selon un code spécifique), envoi du tweet; 3 . Réception des twoutils, correction individuelle et collective, dictée d'évaluation (facultative); 4 . Dictée de transfert (facultative).

${ }^{7}$ Ludovia : http://ludovia.org/

${ }^{8}$ https://www.twictee.org/2016/12/22/vous-prendrez-bien-un-cafetwictee/

9 Twittconte, TweetenRimes, Twoulipo, Clément Aplati...

${ }^{10}$ https://www.twictee.org/2015/07/27/la-charte-du-twictonaute/
} 


\section{Méthodologie}

Dans le cadre du projet de recherche "Twictée pour apprendre l'orthographe " ${ }^{11}$, quarante classes d'écoles et collèges, de contextes sociaux et géographiques divers, proposées par l'association et par les inspections partenaires (académies de Créteil et de Grenoble), ont participé à l'étude. Dix-neuf d'entre elles utilisaient le dispositif Twictée. Cette recherche est structurée en deux volets : le premier porte sur l'enseignement et l'apprentissage de l'orthographe, le second sur le développement professionnel des enseignants. Cet article s'inscrit dans ce deuxième axe.

\section{Les participants}

Les enseignants que nous avons pu interviewer, et dont les classes ont par ailleurs été observées, enseignent en quatrième et cinquième année de l'école élémentaire (CM1 et CM2) pour dix-sept d'entre eux et en sixième année ( $6^{\mathrm{e}}$ de collège) pour les deux autres. Leur expérience dans le dispositif Twictée est variable: neuf débutent, cinq s'engagent pour la seconde année et cinq ont plus de trois ans d'expérience. Parmi ces derniers, deux ont participé au dispositif dès son lancement et sont des membres actifs de l'association. Tous se sont portés volontaires pour participer à cette recherche à la suite de notre invitation relayée par l'association Twictée ou leur inspecteur.

\section{Les entretiens}

En 2017, vingt enseignants avaient initialement donné leur accord pour participer à cette recherche. Un d'entre eux s'est rapidement désisté, un autre n'a pas pu être interviewé en début d'année et cinq n'ont pas répondu aux demandes d'entretiens la seconde année. L'analyse porte donc sur cinquante et un entretiens (18 en octobre 2017, 19 en juin 2018 et 14 en juin 2019). Il est à noter que parmi les quatorze enseignants interrogés en 2019, cinq n'ont pas effectué de twictées durant l'année scolaire. Les entretiens, semi-directifs d'une durée d'une heure, ont porté sur les motivations des enseignants à participer à ce dispositif, sur l'intensité et la nature de leur participation et sur les changements qu'ils ont perçus, en relation avec cette participation.

\section{Analyse des entretiens}

Pour analyser nos données, nous nous sommes inspirés des travaux de Daele (2013), qui mettent en évidence les liens entre le dispositif (les modes d'organisation de la communauté et les opportunités d'apprentissage qu'elle offre) et les dynamiques individuelles de ses membres (la manière dont ils se saisissent ou non de ces opportunités en fonction de leur participation et de leur engagement), et de ceux de Lameul, Peltier et Charlier (2014), qui mettent en relation l'engagement, les changements de pratiques et le développement professionnel.

Ainsi, pour comprendre la relation entre le dispositif, la participation de ces enseignants et les effets éventuels sur leur développement professionnel, nous avons procédé à une analyse qualitative. Nous avons relevé dans les discours des enseignants :

\footnotetext{
${ }_{11}$ Cette recherche a été financée dans le cadre des projets e-FRAN (Espaces de formation, de recherche et d'animation numérique, Programme d'Investissement d'Avenir 2). Voir: https://inspe.u-pec.fr/recherche-et-innovation/recherche-et-internationalisation/contrats-et-projets-derecherche/tao-renforcer-les-competences-en-orthographe-avec-le-dispositif-twictee
} 
- la motivation initiale pour entrer dans le dispositif et pour y rester;

- la participation (nombre d'épisodes de twictées ${ }^{12}$ suivis, participation ou non à l'élaboration des twictées);

- le niveau d'engagement (interactions et collaboration avec des pairs, utilisation des ressources produites par l'association, suivi de formation);

- les transformations engendrées par la participation au dispositif du point de vue des acteurs sur le plan des savoirs (acquisition de connaissances dans le domaine de l'orthographe et de son enseignement), des savoirs pédagogiques (changements dans la mise en activité des élèves, dans la manière d'évaluer les élèves), de l'identité professionnelle (plus grande confiance en soi, plaisir retrouvé à enseigner), de l'engagement dans le métier (investissement dans des collectifs professionnels et/ou dans la formation en relation avec la participation au réseau Twictée), et de l'attention portée à la réussite des élèves.

\section{Résultats}

\section{La participation et l'engagement des enseignants interrogés}

Les effets d'un dispositif sur le développement professionnel sont conditionnés par la participation et l'engagement des membres (Daele, 2013; Lameul, Peltier et Charlier, 2014). Ainsi, nous observons, à partir des entretiens, la participation à l'élaboration des twictées (interactions entre enseignants) et aux épisodes proposés en 2017-2018 et 2018-2019 (interactions entre classes), ainsi que l'engagement durant cette période. Nous distinguons ainsi la participation (aux épisodes des twictées) et l'engagement (interactions et collaborations avec des pairs en présence et à distance, utilisation des ressources produites par l'association, investissement dans la formation) qui nécessite une implication plus importante.

\section{LA PARTICIPATION}

En 2017-2018, cinq des enseignants interrogés ont participé aux échanges relatifs aux choix des twictées ${ }^{13}$ $(E 12,14,23,44$ et 47$)$. Seize enseignants sur dix-neuf ont réalisé au moins quatre des six twictées proposées, trois classes n'ont effectué que deux (E19) ou trois (E20 et 48) twictées.

En 2018-2019, quatre enseignants sont intervenus dans l'élaboration des twictées (E11, 17, 45 et 47 ) et seuls sept enseignants ont participé au dispositif (parmi les douze enseignants qui n'ont pas participé, quatre d'entre eux n'ont pas accordé d'entretien en 2019).

\section{L’ENGAGEMENT}

Douze enseignants déclarent avoir eu des interactions en présence sur le dispositif Twictée en 2017-2018, 10 en 2018-2019 (il est à noter que trois enseignants ont gardé le principe de la twictée au sein de leur classe sans participer au dispositif en ligne). Ils ne sont que quatre à déclarer avoir échangé à distance en 2017-2018 et six en 2018-2019. Deux enseignantes déclarent préférer échanger à distance (E15 et 46$)$.

\footnotetext{
${ }_{12} \mathrm{Au} 1 / 12 / 2020$, Twictée en est à l'épisode 47 de la saison 8 . Une saison correspond à une année scolaire et environ six épisodes sont proposés par année. Un épisode se déroule en quatre phases (voir note 6).

${ }^{13}$ Cette phase est la plus propice aux interactions entre enseignants. En amont d'un épisode de la twictée, les enseignants élaborent et choisissent la dictée qui sera travaillée. Cette phase se déroule via un document partagé, les enseignants réalisent un bilan de la séance précédente, décident des notions à travailler puis choisissent une phrase en fonction d'un thème. À chaque épisode, cinq twictées différentes sont proposées selon le niveau de classe.
} 
Dans le travail avec les collègues, c'est un peu difficile en toute honnêteté dans mon établissement, c'est difficile. C'est pour ça que j'apprécie [le réseau] Twictée c'est parce que du coup, j'échange énormément avec des collègues, à partir de Twitter, j'ai un grand réseau de collègues que je n'ai jamais vus physiquement, que je n'ai jamais rencontrés. On suit nos comptes et on échange, quand j'ai des difficultés on s'envoie un message privé. (E15)

Cinq enseignants déclarent avoir suivi des formations sur l'orthographe, dont deux directement par des membres de Twictée.

J'ai eu la chance de rencontrer $M$ [cadre de l'association, devenue conseillère pédagogique] qui travaillait sur ma circonscription et du coup, j'ai pu avoir, mais à titre personnel, une formation complémentaire. (E11)

Onze enseignants font référence aux outils créés par l'association (en particulier le \#DicoBalises). Cinq d'entre eux citent les chercheurs qui ont inspiré la démarche Twictée ${ }^{14}$.

\section{Les changements perçus}

Les changements perçus par les enseignants apparaissent directement liés à la participation et à l'engagement. Quatre profils se distinguent :

\section{Profil 1 : Participation faible, pas d'engagement (E13, 14, 19, 20 et 48)}

Ces enseignants n'ont participé qu'une année et à une partie seulement des épisodes proposés. Ils n'interviennent pas dans le choix de la phrase et interagissent peu entre pairs. Lors de l'entretien de juin 2018, ils n'indiquent aucun changement particulier dans leur pratique.

Je n'ai pas attendu Twictée pour faire de la dictée négociée. (E20)

Profil 2 : Participation forte sur une courte durée, engagement modéré (E12, E15, 17, 23, 43, 44 et 50)

Ces enseignants n'ont participé que la première année de la recherche, mais à l'ensemble des épisodes proposés. Ils se sont approprié les outils indispensables (le \#DicoBalises) et ont interagi en présence ou à distance principalement sur les aspects organisationnels du dispositif. Les changements perçus sont principalement relatifs aux compétences informatiques et au travail en réseau. Tous ces enseignants (sauf E12) déclarent utiliser régulièrement Twitter grâce au dispositif Twictée. L'enseignante E15 émet initialement de fortes réserves sur l'usage du numérique et en particulier sur Twitter. Lors du dernier entretien (2019), elle indique que grâce à Twictée, elle a découvert Twitter et depuis, elle échange régulièrement sur ses pratiques dans des réseaux d'enseignants, elle est même devenue modératrice d'un réseau.

En dehors de la maîtrise des outils, les autres changements déclarés sont moins nombreux. E23 dit avoir adopté une évaluation plus positive, E44 déclare favoriser le travail en petits groupes et la négociation entre élèves. E23 et E44 considèrent être plus efficaces dans leur enseignement. Les modifications sont rarement didactiques. Les enseignantes E43 et E50 travaillent en co-enseignement. Elles notent des changements dans leur manière d'enseigner l'orthographe (utilisation d'un vocabulaire plus précis, recours plus systématique à l'explicitation écrite). Elles n'ont utilisé le dispositif que la première année, mais elles en gardent le principe la seconde année et l'adaptent en fonction de nouvelles modalités pédagogiques visées.

\footnotetext{
${ }^{14}$ L'association Twictée se réfère explicitement aux travaux sur l'orthographe de Brissaud, de Cogis et de Fayol.
} 
Pour qu'elle [la twictée] réponde aux besoins du moment de mes élèves en fait, parce que je suis plutôt partie sur placer mes élèves en condition d'auteur, donc ce sont eux qui créent leurs twictées en fait. (E50 juin 2019)

\section{Profil 3 : Participation forte et continue, engagement modéré (E21 et 22)}

Ces deux enseignantes débutent dans le réseau Twictée, elles interagissent avec des collègues en présence, mais pas à distance. Elles sont toutes les deux très positives sur les apprentissages de leurs élèves. Elles déclarent quelques changements relatifs à leur enseignement de l'orthographe, qui devient plus flexible (E21) ou plus structuré (E22), et une amélioration sensible de leurs compétences dans la maîtrise des outils numériques.

\section{Profil 4 : Participation forte, engagement fort (E11, 16, 45, 46 et 47)}

Ce sont des enseignants qui effectuent la majorité des épisodes, qui participent le plus souvent au choix des twictées, qui connaissent les références didactiques propres au dispositif Twictée et qui s'investissent dans les échanges en présence, mais aussi à distance. Ces enseignants sont ceux qui déclarent les changements les plus nombreux qui touchent à la fois leur pratique, mais aussi (et surtout) leur identité professionnelle.

L'enseignante E45 s'inscrit en 2016 et s'investit rapidement dans les échanges.

Dans le travail avec les collègues, c'est un peu difficile en toute honnêteté dans mon établissement, c'est difficile. C'est pour ça que j'apprécie Twictée, c'est parce que du coup j'échange énormément avec des collègues, à partir de Twitter. (E45 octobre 2017)

En 2017-2018, elle s'engage dans des interactions en présence (notamment dans le comité de suivi du réseau Twictée mis en place par l'académie de Grenoble) et à distance via Twitter.

Je suis allée au comité de suivi, j'ai pu parler de mes pratiques, ça m’a permis de réaliser que jusqu'à maintenant je ne travaillais pas assez l'orthographe; il y a le réseau Twitter donc un groupe énorme d'enseignants qui participent au dispositif, du coup tu discutes avec énormément d'enseignants, ça franchement, ça m'a beaucoup apporté; on n'a pas arrêté d'échanger à la fois par Twitter et ensuite de se téléphoner pour mettre en place nos séquences. (E45 juin 2018)

En 2018-2019, elle s'investit de plus en plus dans le réseau Twictée et dans d'autres projets créés par des membres de la communauté (\#twittenRime, \#twoulipo), elle participe pour la première fois au choix des twictées et anime des formations. Elle exprime son plaisir à enseigner parce qu'elle dit évoluer dans ses pratiques et se sentir plus efficace avec ses élèves.

L'adhésion à l'association joue un rôle important dans le processus d'interaction entre pairs.

L'enseignante E11 participe au réseau Twictée pour la seconde année en 2017-2018. Elle effectue tous les épisodes, mais intervient peu dans le choix des twictées, car elle ne se sent pas suffisamment légitime. Cependant, elle discute en présence avec des formateurs engagés dans Twictée, découvre Twitter et suit les comptes personnels des responsables de l'association, ce qui suscite sa curiosité et favorise une certaine prise de risque (Lantz-Andersson, Lundin et Selwyn, 2018).

Ça m'a montré que j'étais capable de jongler entre plusieurs notions en même temps, passer de quelque chose de très structuré, finalement rassurant [...], à quelque chose de plus ouvert. (E11 juin 2018)

En septembre 2018, elle décide de « franchir le pas ». Elle adhère à l'association. 
Je vous avoue que là en basculant, en fait oui, dans ce monde, vous voyez, dans ce club on va dire, j'ai effectivement accès aux coulisses pédagogiques, quelque chose qui renforce ce qui se passe après sur le côté préparation des twictées, au niveau de la construction même du dispositif. (E11 juin 2019)

Elle s'investit dans la préparation des twictées, prend confiance et participe aux échanges sur les slacks de l'association, ce qui enrichit, selon elle, sa réflexion didactique.

Ça fait dix ans que je suis ici et ça fait dix ans que c'est les mêmes dix collègues et sur Twitter, voilà on a des échanges avec des gens d'environnements différents, professeurs de collège, etc. On a un sentiment de confiance et de remise en question par rapport à nos pratiques qui aboutit toujours, qui donne des solutions sur certaines choses... Sur les slacks, on est liés parce qu'on partage une certaine conviction, les échanges sont orientés sur ce qui nous a réunis, c'est beaucoup plus intéressant parce qu'on va tous dans la même direction... II y a moins de perte de temps de dispersion, de critique, il y a des contradictions, mais j'ai l'impression qu'elles sont moins dans la critique et elles sont là pour avancer, il y a de la bienveillance. (E11 juin 2019)

Les enseignants qui exercent des responsabilités dans l'association déclarent des changements identitaires importants. Ainsi l'enseignant E16, directeur d'école, s'est d'abord impliqué dans le réseau Twictée à titre personnel puis il a peu à peu mobilisé son école, il est ensuite devenu plombier ${ }^{15}$, avant de devenir président de l'association en 2019; il évoque de nombreux changements relatifs à la confiance en soi et au sentiment de compétence.

J'ai grandi pédagogiquement, j'ai utilisé des outils que je n'aurais jamais utilisés de ma vie, moi qui suis juste enseignant dans ma classe, je me retrouve à présenter le dispositif à des inspecteurs, j'ai créé \#tweetconte avec R.; j'ai pris des degrés de compétence dans tout ce qui est gestion de communauté, j'ai pris aussi de la compétence sur les différents outils tableur sur et même sur être bienveillant avec les collègues. (E16 octobre 2017)

L'enseignante E47 s'est engagée très tôt dans le réseau, elle fait aujourd'hui partie du conseil d'administration et participe à la formation des nouveaux inscrits. Elle évoque une conception collaborative du métier.

Le dispositif, c'est tellement plus que l'orthographe, c'est aussi tout ce qui nous permet de faire de la formation de nous former nous-mêmes et de former d'autres, voilà on fait quelque chose ensemble et chacun a une valeur dans un travail collaboratif. (E47)

Ainsi, les changements déclarés par les enseignants les plus investis, essentiellement les adhérents de l'association, touchent aux différents aspects du développement professionnel, aux savoirs disciplinaires ( $E 11, E 45$ et 46), à la réussite des élèves ( $E 46$ met en avant le rôle de l'écrit dans le dispositif Twictée qui favorise, selon elle, l'accès aux représentations et la réussite de ses élèves) et surtout à l'engagement dans le métier et au renforcement de l'identité professionnelle. Ces changements sont d'autant plus significatifs, selon les enseignants (E16, E45, E46, E47), lorsqu'ils sont amenés à présenter le dispositif ou à organiser des formations, ce qui les amène à conceptualiser, à expliciter et à justifier la démarche didactique du dispositif.

\footnotetext{
${ }^{15}$ Ce sont les membres de l'association qui s'assurent que tout fonctionne. Ils gèrent notamment les inscriptions, les appariements des classes et règlent tous les incidents techniques.
} 


\section{Discussion}

Le premier élément qui apparaît à la suite de cette analyse, c'est la faiblesse de la participation sur la durée des enseignants observés. Ils s'étaient pourtant engagés, auprès de l'équipe de recherche, à participer au dispositif durant les deux années de suivi.

Lameul, Peltier et Charlier (2014) indiquent que, pour comprendre l'engagement des acteurs, il est nécessaire d'interroger leur motivation, leur niveau de satisfaction et leurs intentions de poursuivre leur participation. Comment dès lors comprendre un tel taux d'abandon, puisque lors des entretiens menés en fin de première année, tous les acteurs déclarent leur satisfaction vis-à-vis du dispositif Twictée, pour leurs élèves, mais aussi pour eux-mêmes, ainsi que, à une exception près, leur intention de poursuivre l'année suivante?

C'est motivant, c'est plus dynamique et pour nous ça redonne un peu la pêche pour l'enseignement de l'orthographe et on voit par rapport aux élèves, c'est plus réactif dans les apprentissages. (E14)

Je me suis rendu compte de la richesse des manières de travailler, des manières de coller la twictée à son projet de classe aussi c'était vraiment intéressant. (E48)

Ces défections concernent aussi bien les débutants ( 6 sur 9 ) que les plus expérimentés ( 3 sur 5 pour ceux qui ont trois ans d'ancienneté en juin 2018, 2 sur 5 pour quatre ans).

De multiples raisons sont invoquées pour justifier cet abandon : une mutation sur un autre poste ( $E 19$ et 23), la participation à d'autres projets (E15 et 48). E17 explique son désinvestissement par le non-respect des règles par les classes partenaires (elle n'a pas reçu les productions des autres classes). E43 et 50 trouvent les contraintes trop fortes, elles gardent le principe entre leurs deux classes et demandent aux élèves d'élaborer eux-mêmes les twictées. E12 et 44 participent depuis les débuts du réseau Twictée, ils se plaignent tous les deux de la forte augmentation des classes participantes.

De même, peu d'enseignants s'engagent dans les discussions relatives au choix des twictées. Les obstacles, déjà identifiés par la recherche, sont principalement liés à l'ancienneté (Audran et Simonian, 2009) et à la taille de la communauté (Drot-Delange, 2001).

Les nouveaux arrivants ne se sentent pas assez reconnus ou légitimes.

J'ai essayé de participer, mes suggestions n'ont pas été retenues donc ça ne m'a pas motivé à insister, le cycle trois était vraiment très nombreux c'était assez fastidieux (E46). Ce que j'aimerais bien faire, c'est la phase d'élaboration où il faut préparer le texte avec d'autres collègues, je le fais pas encore moi ça, participer au texte collaboratif en fait ça je n'ai pas encore assez de recul pour réussir à le faire, mais c'est mon objectif pour la suite, je pense qu'il faut d'abord rentrer dans le dispositif Twictée et comprendre bien comment ça fonctionne. (E21)

Les anciens de Twictée déplorent l'explosion des effectifs.

Au début, j'étais extrêmement investie dans la phase de préparation, sauf que maintenant, je trouve qu'il y a beaucoup beaucoup de monde et se mettre d'accord avec dix, vingt personnes 
c'était possible, maintenant quand notre document de préparation fait 35 pages et que dedans il y a cent et quelques personnes... pour moi c'est trop quoi. (E44)

Les interactions avec les classes miroirs, les classes partenaires à qui il faut envoyer les productions, pourraient donner lieu à des collaborations entre enseignants, mais dans la réalité ces échanges restent inexistants ou superficiels.

Ça fait très Meetic, on est content de voir plein de monde, mais derrière on ne crée aucun lien. (E12)

Comment expliquer l'absence d'interactions entre les partenaires alors que celles-ci sont au cœur de ce projet d'enseignement collaboratif d'enseignement? Daele (2013) ainsi que Lameul, Peltier et Charlier (2014) soulignent l'importance de la motivation pour expliquer l'adhésion au dispositif. L'entrée dans le dispositif Twictée s'explique par la volonté des enseignants d'offrir un enseignement plus motivant de l'orthographe pour les élèves et pour eux-mêmes. Du côté des élèves, ils mettent en avant le caractère ludique qu'ils prêtent au dispositif et la présence du numérique censée motiver les élèves (Cadet, Crinon et Ferone 2019). En ce qui les concerne, innover leur permet de retrouver du plaisir à faire leur métier.

Les enfants adorent vraiment, les enfants adorent pourtant c'est de l'orthographe, mais ils ont l'impression que c'est un outil moderne de leur génération donc ça les captive (E22). Pour changer, enfin pas m'obliger, mais pour aller voir ailleurs comment faire autrement, pour me remotiver en fait. (E14)

En fait, seuls les membres les plus expérimentés, et en particulier les adhérents de l'association, évoquent le collectif et l'importance des interactions entre les participants.

Ce constat amène à s'interroger sur les typologies proposées par les recherches antérieures concernant les réseaux et communautés d'enseignants. La distinction qui oppose les réseaux, où des enseignants donnent à voir leurs pratiques, exposent des idées et d'autres les consomment sans véritable collaboration, aux communautés d'apprentissage professionnelles, où les enseignants construisent et analysent collectivement leur enseignement, est utile pour étudier les collectifs enseignants. Mais cette distinction ne permet pas, à elle seule, d'envisager les effets de la participation sur le développement professionnel. Ce qu'il importe de regarder, c'est le rapport des participants au collectif, rapport évolutif et différencié d'un participant à l'autre. Dans le cas de Twictée, pour la grande majorité des enseignants, il s'agit de participer à un réseau de classes, à un dispositif qu'ils considèrent ludique et motivant pour les élèves et pour eux-mêmes. Les changements de pratiques sont en cohérence avec les motifs d'engagement et correspondent aux conceptions dominantes actuelles de l'enseignement (Crinon et Ferone, 2018; Ferone, 2019). II s'agit de favoriser la parole entre élèves, de leur proposer des démarches de construction, de les motiver par des situations ludiques, en ayant souvent recours au numérique pour cela, et de les évaluer positivement.

À l'inverse, une minorité, principalement les membres de l'association, adhèrent à une communauté. II s'agit pour ceux-là d'animer un collectif, d'apporter aux autres un soutien logistique, mais également pédagogique, ce qui implique de se former, de construire collectivement des outils et des ressources, de négocier ensemble les objectifs, les règles de fonctionnement et de participation de la communauté. Chez eux, les changements peuvent être pédagogiques et didactiques, mais ils sont surtout identitaires (meilleure estime d'eux-mêmes, sentiment d'être reconnus...). 


\section{Conclusion}

Alors que les communautés en ligne d'enseignants se développent fortement depuis l'apparition des réseaux sociaux et que l'intérêt de la communauté scientifique pour ce phénomène est croissant, nous nous sommes intéressés à un collectif francophone particulièrement dynamique, Twictée. Communauté qui se déclare engagée dans "la réussite de tous les élèves et le développement professionnel des enseignants ${ }^{16} »$.

Pour l'étudier, nous nous sommes appuyés sur la distinction entre deux catégories de collectifs connectés : les réseaux et les communautés d'apprentissage professionnelles (Wenger, Trayner et De Laat, 2011). Nos analyses montrent que cette classification est utile pour observer le fonctionnement du collectif, mais qu'elle ne permet pas d'identifier les effets sur le développement professionnel des individus. Non seulement il apparaît nécessaire d'observer les dynamiques des collectifs sur la durée (Beauné et al., 2019), mais aussi (et surtout) celles des trajectoires individuelles de ses membres.

Dans le cas du réseau Twictée, nous avons mis en évidence quatre profils de participation et d'engagement avec pour conséquence des effets variables sur le développement professionnel. Ce qui semble déterminant, c'est l'objectif de participation que se fixent les membres.

La motivation initiale des twictonautes (ceux qui participent au dispositif Twictée) est liée à l'enseignement de l'orthographe; il s'agit surtout de moins s'ennuyer et de moins ennuyer les élèves dans un enseignement, celui de l'orthographe, parfois considéré comme difficile ou rébarbatif. Le dispositif Twictée propose, de leur point de vue, une façon ludique et attractive d'apprendre l'orthographe, grâce au numérique et aux échanges entre classes. Pour ces enseignants, l'essentiel des interactions est centré sur les aspects matériels et organisationnels, et les changements indiqués sur les pratiques sont d'ordre pédagogique (la discussion et le travail de groupe sont favorisés, les évaluations sont plus positives). Une minorité, ceux qui s'interrogent sur les fondements didactiques du dispositif Twictée, témoignent de transformations plus importantes (travail de catégorisation, élaboration des justifications à l'écrit).

Ce qui semble constituer une étape importante dans les parcours observés est l'adhésion à l'association. Elle permet pour les nouveaux membres de participer, d'interagir en toute sécurité avec les autres membres dans les canaux de discussion privée de l'association, ce qu'ils n'osent pas faire sur Twitter où les discussions pourraient devenir virulentes. Pour les plus anciens membres de l'association, l'exercice de responsabilités entraine des effets importants sur l'identité professionnelle, notamment lorsqu'ils forment les nouveaux arrivants ou qu'ils présentent le dispositif à des supérieurs hiérarchiques.

En fait, deux collectifs fonctionnent en parallèle; le premier, les twictonautes, regroupe les enseignants qui interagissent avec leur classe pour effectuer les différents épisodes des twictées, le second regroupe les membres de l'association, qui participent ou non aux twictées, qui font fonctionner le dispositif et qui interagissent sur de nombreux autres sujets : l'association propose ainsi plus d'une trentaine de canaux de discussion (slack) relatifs à des sujets propres au réseau Twictée ou à des sujets plus généraux sur la pédagogie, le développement de carrière...

Ainsi, pour certains, Twictée constitue un réseau qui procure des activités pour les élèves, des ressources et des personnes-ressources qu'ils utilisent pendant un certain temps avant de changer à nouveau leurs manières de faire. Pour d'autres à l'inverse, il s'agit de s'impliquer pleinement dans une communauté, d'en partager les valeurs et de participer à son destin. Nous avons précédemment qualifié Twictée de communauté d'apprentissage professionnelle, mais il conviendrait sans doute de réserver ce qualitatif aux

\footnotetext{
${ }^{16}$ https://www.helloasso.com/associations/twictee
} 
membres de l'association. Pour mieux comprendre les effets de l'engagement des acteurs les plus engagés dans une communauté, nos prochaines analyses porteront spécifiquement sur les membres de l'association.

\section{Liste de références}

Audran, J. et Simonian, S. (2009). Étudier les communautés d'apprenants en ligne : quel(s) agencement(s) des méthodes de recherche? Éducation \& Formation, e-290, 7-18. http://revueeducationformation.be/index.php?revue=6\&page=3

Baron, G.-L. et Zablot, S. (2017). De la constitution de ressources personnelles à la création de communautés formelles : étude de cas en France. Review of science, mathematics and ICT education, Laboratory of Didactics of Sciences, Mathematics and ICT, Department of Educational Sciences and Early Childhood Education - University of Patras, 11(2), 27-45. https://hal.archives-ouvertes.fr/hal-01671526

Beauné, A., Levoin, X., Bruillard, É., Quentin, I., Zablot, S., Carton, T., Rouvet-Song. C., Normand-Assadi. S., Le Roy. M., Nikishina, T., Mas-Costesèque. S. et Louis Baron, G. (2019). Collectifs en réseau d'enseignants producteurs de ressources. Rapport scientifique des laboratoires STEF et EDA dans le cadre de la convention DNE. [Rapport de recherche] Université Paris 5 Sorbonne Descartes; ENS Cachan. https://hal.archives-ouvertes.fr/hal-02022830

Bergviken Rensfeldt, A., Hillman, T. et Selwyn, N. (2018). Teachers 'liking' their work? Exploring the realities of teacher Facebook groups. British Educational Research Journal, 44(2), 230-250.

Cadet, L., Crinon, J. et Ferone, G. (2019). Former au raisonnement orthographique. Conceptions d'enseignants du cycle 3. Repères, 60, 153-171. https://doi.org/10.4000/reperes.2371

Caviale, O. et Bruillard, É. (2009). Les jeux d'acteurs sur des listes de discussion institutionnelles d'enseignants. Réseaux, 155(3), 137-176. https://doi.org/10.3917/res.155.0137

Clot, Y. (2005). L'auto-confrontation croisée en analyse du travail : l'apport de la théorie bakhtinienne du dialogue. Dans L. Filliettaz et J.-P. Bronckart (dir.), L'analyse des actions et des discours en situation de travail (p. 37-55). Louvainla-Neuve : Peeters.

Crinon, J. et Ferone, G. (2018). Savoirs et conceptions professionnelles des enseignants. Éducation \& Formation, Les enseignants et leur métier : entre doxas et incertitudes, e-310, 39-57. http://revueeducationformation.be/index.php?revue $=30$

Champy-Remoussenard, P. (2014). Des dimensions collectives comme dimensions génériques du travail humain et leurs déclinaisons dans des activités d'enseignement partenariales. Questions vives, 21. https://doi.org/10.4000/questionsvives. 1534

Daele, A. (2013). Discuter et débattre pour se développer professionnellement : analyse compréhensive de l'émergence et de la résolution de conflits sociocognitifs au sein d'une communauté virtuelle d'enseignants du primaire [thèse de doctorat, Université de Genève]. https://archive-ouverte.unige.ch/unige:27065

Dionne, L., Lemyre, F. et Savoie-Zajc, L. (2010). Vers une définition englobante de la communauté d'apprentissage (CA) comme dispositif de développement professionnel. Revue des sciences de l'éducation, 36(1), 25-43.

Drot-Delange, B. (2001). Outils de communication électronique et disciplines scolaires : quelle(s) rationalité(s) d'usage? Le cas de trois disciplines du second degré : la technologie au collège, l'économie-gestion et les sciences économiques et sociales au lycée [thèse de doctorat en sciences de l'éducation, ENS de Cachan]. https://tel.archives-ouvertes.fr/tel-00381040/document

Dupriez, V. (2015). Le point de vue des travaux sur l'organisation des établissements scolaires. Dans L. Ria (dir.), Former les enseignants au XXIe siècle, volume 1 : Établissement formateur et vidéoformation (p. 49-59). Louvain-la-Neuve : De Boeck Supérieur.

Ferone, G. (2019). Numérique et apprentissages : prescriptions, conceptions et normes d'usage. Recherches en éducation, 35, 63-75. https://doi.org/10.4000/ree.1312

Henri, F. et Pudelko, B. (2002). La recherche sur la communication asynchrone : de l'outil aux communautés. Dans A. Daele et B. Charlier (dir.), Les communautés délocalisées d'enseignants, Programme Numérisation pour l'Enseignement et la Recherche (PNER), 12-48, Paris.

Kaddouri, M. (2011). Motifs identitaires des formes d'engagement en formation. Savoirs, 25(1), 69-86.

Koschmann, T. (dir.) (1996). CSCL: Theory and practice of an emerging paradigm. Mawhwah, NJ: LEA. 
Laferrière, T. (2001). Teacher learning, community and technology. International symposium on learning communities, Barcelona, Spain.

Lameul, G., Peltier, C. et Charlier, B. (2014). Dispositifs hybrides de formation et développement professionnel : effets perçus par des enseignants du supérieur. Éducation \& Formation, e-301, 99-113. http://revueeducationformation.be/index.php?revue=19

Lantheaume, F. (2016) La professionnalité des enseignants à l'épreuve de la durée: les ressources de la plasticité professionnelle. Dans L. Ria (dir.), Former les enseignants au XXI siècle, Volume 2: Professionnalité des enseignants et de leurs formateurs (p. 67-75). Louvain-la-Neuve : De Boeck Supérieur.

Lantz-Andersson, A., Lundin, M. et Selwyn, N. (2018). Twenty years of online teacher communities: A systematic review of formally-organized and informally-developed professional learning groups. Teaching and Teacher Education, 75, 302-315.

Leclerc, M. et Labelle, J. (2013). Au cœur de la réussite scolaire : communauté d'apprentissage professionnelle et autres types de communautés. Éducation et francophonie, 41(2), 1-9.

Liu, K., Miller, R. et Jahng, K. E. (2016). Participatory media for teacher professional development: Toward a self-sustainable and democratic community of practice. Educational Review, 68(4), 420-443.

Macià, M. et García, I. (2016). Informal online communities and networks as a source of teacher professional development: A review. Teaching and Teacher Education, 55, 291-307.

Marcel, J.-F. (2005). Apprendre en travaillant. Contribution à une approche sociocognitive du développement professionnel de l'enseignant. Université de Toulouse II - Le Mirail.

Pastré, P., Mayen, P. et Vergnaud, G. (2006). La didactique professionnelle. Revue française de pédagogie, 154, $145-198$.

Peters, M. et Savoie-Zajc, L. (2013). Vivre une CAP : appréciations de participants sur les retombées professionnelles perçues. Éducation et francophonie, 41(2), 102-122.

Quentin, I. (2014). Fonctionnements et trajectoires des réseaux professionnels en ligne : le cas des réseaux d'enseignants. Distance et médiations des savoirs, 7. https://doi.org/10.4000/dms.815

Quentin, I. (2019). Pratiques de communication d'enseignants sur Twitter et collectifs. Dans A. Beauné et al. (dir.), Collectifs en réseau d'enseignants producteurs de ressources. Rapport scientifique des laboratoires STEF et EDA dans le cadre de la convention DNE [Rapport de recherche]. Université Paris 5 Sorbonne Descartes; ENS Cachan. https://hal.archives-ouvertes.fr/hal-02022830

Rayou, P. (2018). Savoirs et conceptions professionnelles des enseignants. Éducation \& Formation, e-310. http://revueeducationformation.be/index.php?revue $=30$

Sésamath. (2020). Profession de foi de l'association. http://www.sesamath.net/index.php?page=professiondefoi

Scardamalia, M. et Bereiter, C. (1994). Computer support for knowledge-building communities. Journal of the Learning Sciences, 3(3), 265-384.

Tardif, M., Lessard, C. et Lahaye, L. (1991). Les enseignants des ordres d'enseignement primaire et secondaire face aux savoirs. Esquisse d'une problématique du savoir enseignant. Sociologie et société, 23(1), 55-69.

Trust, T., Krutka, D. G. et Carpenter, J. P. (2016). Together we are better: Professional learning networks for teachers. Computers \& Education, 102, 15-34.

Uwamariya, A. et Mukamurera, J. (2005). Le concept de « développement professionnel » en enseignement : approches théoriques. Revue des sciences de l'éducation, 31(1), 133-155.

Vangrieken, K., Meredith, C., Packer, T. et Kyndt, E. (2017). Teacher communities as a context for professional development: A systematic review. Teaching and teacher education, 61, 47-59.

Wells, C. et Feun, L. (2007). Implementation of learning community principles: A study of six high schools. NASSP Bulletin, $91,141-160$.

Wenger, E. (1998). Communities of practice: Learning, meaning, and identity. Cambridge, UK: Cambridge University Press.

Wenger, E., Trayner, B. et De Laat, M. (2011). Promoting and assessing value creation in communities and networks: $A$ conceptual framework. Heerlen, the Netherlands: Ruud de Moor Centrum, Open University. 